Open Access

\title{
A tractable approach to analyzing the energy-aware two-way relaying networks in the presence of co-channel interference
}

\author{
Dinh-Thuan Do and Hoang-Sy Nguyen * (D)
}

\begin{abstract}
Under the impact of co-channel interference $(\mathrm{CCl})$ at two source nodes and the relay, we investigate the performance of dual-hop amplify-and-forward (AF) relaying networks. In terms of energy harvesting, the power-constrained relay first scavenges energy from the received signal and $\mathrm{CCl}$ signals then amplifies them and forwards them to the destination. In particular, we provide closed-form expressions for outage probability and bit error ratio (BER) to easily analyse the system performance. In this paper, the impact of distinct interference power level and the number of CCls are derived. Monte Carlo simulations are used to provide expressions related to outage probability performance. It is confirmed that energy efficiency at the relay can be enhanced due to several advantages of CCI signals which can also prolong the life expectancy of relaying systems.
\end{abstract}

Keywords: Amplify-and-forward (AF), Co-channel interference (CCI), Bit error ratio (BER), Energy harvesting, Time switching, Two-way relaying

\section{Introduction}

Limited lifespan is a big problem of energy-constrained wireless communication networks. As a result, in order to ensure network connectivity, batteries need to be recharged or replaced periodically. Nevertheless, due to its high expenses, instability and complexity, harvesting energy from natural sources such as wind, solar or vibration which helps lengthen the lifetime of wireless communication networks has attracted much research interest. However, energy harvested from those sources depends on several factors, including weather conditions, which result in high requirements for reliable communication systems. Such limitations can be alleviated by harvesting energy from man-made radio frequency (RF) electromagnetic radiation $[1,2]$. Wireless power transfer (WPT) through electromagnetic waves is a prime candidate in terms of energy harvesting $(\mathrm{EH})$ techniques, which is easy to employ at the receiver compared to aforementioned sources. Recently, low-power electronic devices can be greatly supported thanks to the use of RF-EH

*Correspondence: nguyenhoangsy@tdt.edu.vn

Wireless Communications Research Group, Faculty of Electrical \& Electronics Engineering, Ton Duc Thang University, Ho Chi Minh City, Vietnam
$[3,4]$. Because both information and energy can be transmitted, simultaneous wireless information and power transfer (SWIPT) becomes increasingly popular with a huge number of research topics. The authors in $[5,6]$ investigated the balance between capacity and energy. In particular, some protocols, including time switching and power splitting for SWIPT systems, were given [7]. Furthermore, the optimal transmit co-variance and rateenergy region were obtained.

There have been a few works conducted on the performance of dual-hop cooperative networks under the impact of co-channel interference (CCI). The work in [8] focused on the end-to-end performance of multiuser amplify-and-forward (AF) cooperative networks and showed that CCI seriously impairs the relay-destination connection. In the case of average throughput, transmitting directly and over AF relaying systems by adjusting the number of interfering relays and the target signal-tonoise ratio (SNR) generates substantial gains. Regarding AF schemes, the impact of multi-user interference was carefully considered in case interference does not exist, in which according to asymptotic analysis, the diversity gain of the network is limited by interference [9]. The authors in [10] investigated opportunistic relaying under 
the impact of interference and thermal noise in case channel sensing is operated over slow fading environments. In [11], a dual-hop relay fading network was evaluated in terms of the performance of outage probability of AF and decode-and-forward (DF) relaying schemes in environments where less interference is expected, in which CCIs affect negatively the destination node when the relay node suffers from an additive white Gaussian noise (AWGN). Furthermore, the authors in [12] put forward an interference-aidede $\mathrm{EH}$ scheme for DF relaying networks, in which energy is harvested by the relay from the received signal and CCI signals; after that, the received information signal is decoded before being forwarded to the destination. In [13], the performance of multi-antenna two-way relay networks was considered, in which AF and DF relaying strategies wer systemically analysed, and they proposed an antenna selection scheme by optimizing the received SNR.

In principle, CCIs impair the performance of the system which are caused by some sources of interference. Regarding multi-hop relaying, a number of investigations on the impact of CCIs on the performance of the system were conducted. It is assumed that CCIs were subject to Rayleigh fading and the system performance which were evaluated in terms of outage probability, average symbol error probability (ASEP) and ergodic capacity $[14,15]$. Meanwhile, CCIs were assumed to be subject to Nakagami-m fading channels to compute outage probability, error probability and ergodic capacity which were investigated in [16], and over Nakagami-m fading channels, the performance of CSI in AF multi-hop relaying networks under the impact of CCI was considered [17]. However, the performance metrics described above must be considered independently, if CCIs depend on Nakagami-m fading and solving mathematical problems is demanding. Furthermore, providing better practical insights into a tractable technique to investigate the system performance is nearly impossible as per the results in [16-18]. Furthermore, in [19], the authors focused on AF relaying networks based on two protocols: (i) time switching-based relaying (TSR) and (ii) power splittingbased relaying (PSR) for transmitting information and energy from source node to relay node. Meanwhile, in [20], the ergodic achievable secrecy rate (EASR) of multiple-antenna amplify-and-forward relaying networks was investigated, in which the relay can be wiretapped by an eavesdropper.

Unfortunately, in the presence of $\mathrm{CCI}$, the $\mathrm{EH}$ relaying mode has not been comprehensively studied so we change the EH-assisted relay model in [19] to a two-way relay architecture and the impact of $\mathrm{CCI}$ is investigated. Moreover, the impact of CCI on every node in two-way relaying channels leads to the introduction of the fixed time switching regime in the $\mathrm{EH}$ protocol which has not been considered before. Regarding harvesting energy from both sources and CCI signals, we consider an AF relaying network which assists the power-constrained relay in transmitting signals to the destination. The contributions of our paper are described follows.

- We propose an EH protocol which can harvest energy with pre-calculated time switching coefficients from the source signals in two-way relaying networks and CCI to enhance transmission between the $S_{1}$ and $S_{2}$ source pair considering a dual-hop AF relaying network.

- Closed-form expressions for outage probability and throughput are provided for analysing the system performance. In fact, a tractable model for evaluating EH two-way relaying networks is first introduced under the impact of CCI, and hence, such a model is important to satisfy the acceptable QoS threshold for specific system performance.

- For tractable computation, we derive expressions of bit error ratio (BER) in both cases (with CCI and without CCI). These results can be checked easily by popular tools such as Matlab or Mathematica.

- We give numerical results to prove the impact of source power, interference power and the number of CCIs on the performance of outage probability and throughput. Meanwhile, controlling CCIs at acceptable limits can retain the quality of the transmission link, despite using power-constrained relays.

We organized this paper as follows: In Section 2, we introduce the system model and the proposed EH protocol. Expressions for outage probability and throughput of the system are provided, and the closed-form expression of BER is presented in Section 3. In Section 4, numerical results are given to prove analytical expressions. Eventually, Section 5 draws a conclusion for the paper.

Notation: $f_{W}($.$) and F_{W}($.$) stand for the probability$ density function (PDF) and cumulative distribution function $(\mathrm{CDF})$ of the random variable $(\mathrm{RV}) W$, respectively. $\operatorname{Pr}\{$.$\} denotes the probability distribution. Statistical mean$ operation is denoted by $E$ [.].

\section{System model}

In Fig. 1, under the impact of CCI, we illustrate an EH relay-aided cooperative network, where a relay node $R$ communicates with two sources $S_{1}$ or $S_{2}$. Information can be processed by the two source nodes in two time slots, in which in the first time slot, signals are transmitted to the relay at the same time by both sources $S_{1}$ and $S_{2}$ while during the next time slot, the received signal is amplified and then forwarded by the relay and both source nodes can receive the transmitted signal. 


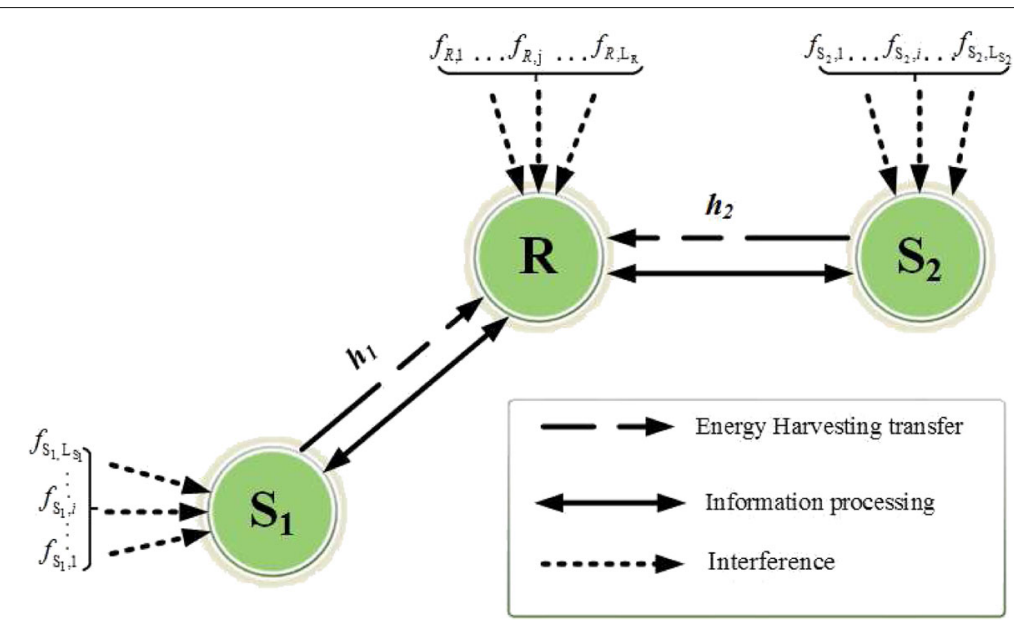

Fig. 1 System model

It is assumed that interference is not only considered as energy in $\mathrm{EH}$ but also noise in information processing. In two-way relaying networks, data flow is assumed to be between source nodes $S_{1}$ and $S_{2}$ in bidirectional transmission. Both nodes $S_{1}$ and $S_{2}$ are powered by a regulated power supply source $E_{S}$ while energy can be harvested by the energy harvesting-assisted relay node by using wireless power based on the energy harvesting protocol. In this model, the received signal and CCI signals are considered as a new source of power for recharging the relay. Additionally, it is noted that each node is equipped with an antenna to increase spatial diversity.

The channel is assumed as block fading, in which the channel remains constant during the transmission of one block and varies from one block to another. In a signal block, $x_{S_{k}}(n)$ denotes the narrow-band transmit signal at $S_{k}, k \in\{1,2\}$ with mean value expressed as $E\left[\left|x_{S_{k}}(n)\right|^{2}\right]=1$, where time index is denoted by $n$ and $d_{R, j}$ denotes as interferer signals, $j$ th, to the relay node with unit mean value. Rayleigh fading channel is at each hop, and $h_{1}$ stands for channel gains between the source $S_{1}$ and the relay node, while $h_{2}$ represents as channel gains between the source $S_{2}$ and the relay node. The channel fading gain between the interferer $i$ th and source $S_{1} ; S_{2}$ is denoted as $f_{S_{1}, i}, f_{S_{2}, i}$ while the channel fading gain between the interferer $j$ th and the relay node is $f_{R, j}$, in which $i=$ $1, \ldots, L_{S}, j=1, \ldots, L_{R}$. For simplicity, it is noted that $L_{S_{1}}=L_{S_{2}}=L_{S}$. Besides that, $E_{S}$ denotes as the transmitted power from the source, and it is equal to the transmit power allocation at two sources. Likewise, equal interference power allocation of various CCIs is denoted by $E_{I}$. Note that the number of CCIs at the relay is denoted by $L_{R}$ while $L_{S_{1}}$ stands for the number of CCIs at the source $S_{1}$, and $L_{S_{2}}$ is the CCIs at the source $S_{2}$.
In fact, there is no data transmitted from $S_{1}$ to $S_{2}$ directly, and time slots are used by the two-hop link with the relay node $S_{1} \rightleftarrows S_{2}$. Hence, the baseband-equivalent discrete time model for the received signal at the relay can be written as

$$
y_{R}=\sqrt{E_{S}} h_{1} x_{S_{1}}+\sqrt{E_{S}} h_{2} x_{S_{2}}+\sqrt{E_{I}} \sum_{j=1}^{L_{R}} f_{R, j} d_{R, j}+n_{R},
$$

in which $n_{R}$ denotes the AWGN components arriving at $R$, with $C N\left(0, N_{0}\right)$. For relay-aided link $S_{1} \rightleftarrows R \rightleftarrows S_{2}$, the amplify-and-forward relay scheme is selected to be employed.

\subsection{Energy harvesting}

Instead of finding some ideal transmission strategies, we consider some relay strategies. In this paper, we carefully evaluate the outage probability of two-hop relaying networks under the impact of $\mathrm{CCI}$ and the energy harvestingassisted relaying channel, in which the benefits of energy harvesting relay-aided cooperative transmission are verified. Furthermore, time switching- and AF-based relaying protocols are put forward.

In Fig. 2, the time switching-based relaying (TSR) protocol [19] includes two phases, energy harvesting and information processing. In the first phase, the harvested energy $E_{h}$ processed in $\alpha T$ (in which $\alpha$ is the time switching fraction and $T$ is the block time) is expressed by

$$
E_{h}=\eta \alpha T\left(E_{S}\left|h_{1}\right|^{2}+E_{S}\left|h_{2}\right|^{2}+E_{I} \sum_{j=1}^{L_{R}}\left|f_{R, j}\right|^{2}\right),
$$

where $0<\eta<1$, which denotes as the energy harvesting conversion coefficient, relying on rectification circuitry. The transmitted power from the relay node $E_{R}$ using 


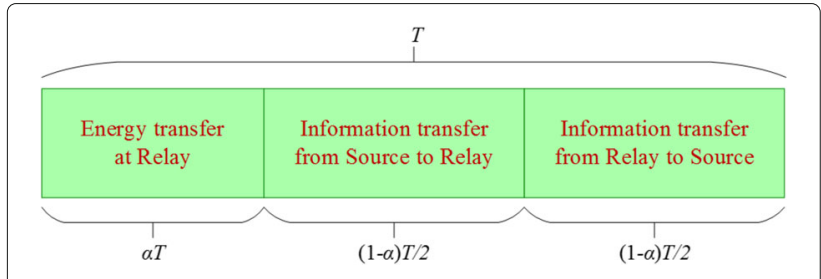

Fig. 2 TSR protocol for energy harvesting

the TSR protocol proposed in [19] can be computed as follows:

$E_{R}=\frac{E_{h}}{(1-\alpha) T / 2}=\rho\left(E_{S}\left|h_{1}\right|^{2}+E_{S}\left|h_{2}\right|^{2}+E_{I} \sum_{j=1}^{L_{R}}\left|f_{R, j}\right|^{2}\right)$,

where $\rho=\frac{2 \eta \alpha}{1-\alpha}$.

In the case of fixed harvesting power at relay, $E_{R}$, time switching coefficients can be calculated as

$$
\alpha_{0}=\frac{E_{R}}{2 \eta\left(E_{S}\left|h_{1}\right|^{2}+E_{S}\left|h_{2}\right|^{2}+E_{I} \sum_{j=1}^{L_{R}}\left|f_{R, j}\right|^{2}\right)+E_{R}} .
$$

As mentioned above, the relay-aided transmission can enhance the amount of power used to transmit signals at the relay. However, the value of instantaneous harvested power flow is time varying, due to the variation of sources of energy. Hence, the power output flow is not as stable as an ordinary power supply. In some cases, there is not enough energy for $R$. Therefore, the minimum amount of energy needed at each relay node to re-transmit signals is the pre-set power level and energy harvested from surrounding sources during a block time.

In principle, the constraint for fixed harvested power using TSR protocol in one-way relaying networks in the case of non-CCI can be expressed as

$$
\frac{\eta \alpha_{T} E_{S}\left|h_{1}\right|^{2}}{\left(1-\alpha_{T}\right) / 2} \leq E_{S}
$$

where time switching fraction is denoted by $\alpha_{T}$ in one-way relaying networks [19].

Next, this equation is equivalent with

$$
\rho E_{S}\left|h_{1}\right|^{2} \leq E_{S}
$$

In this paper, it is noted that under the impact of CCI, the constraint of the energy harvesting policy can be given as

$$
\rho P_{S}\left|h_{k}\right|^{2} \underset{k \in\{1,2\}}{=} \frac{P_{I} \sum_{i=1}^{L_{S_{k}}}\left|f_{S_{k}, i}\right|^{2}+1}{P_{I} \sum_{j=1}^{L_{R}}\left|f_{R, j}\right|^{2}+1} P_{S}
$$

where we denote $P_{S}=E_{S} / N_{0}, P_{I}=E_{I} / N_{0}$.

It is worth noting that $\mathrm{CCI}$ at the relay can contribute to the improvement of the energy harvesting power level at the relay in two-way relaying networks compared with one-way relaying networks. In (7), if the CCI term of $P_{I} \sum_{i=1}^{L_{S_{k}}}\left|f_{S_{k}, i}\right|^{2}$ is greater than $P_{I} \sum_{j=1}^{L_{R}}\left|f_{R, j}\right|^{2}$, the harvested power at the relay can be enhanced compared to the one-way relay regime.

Thus, the fixed time switching coefficient for the proposed energy harvesting protocol in this paper can be re-computed as

$$
\alpha_{1}=\frac{P_{I} \sum_{i=1}^{L_{S_{k}}}\left|f_{S_{k}, i}\right|^{2}+1}{2 \eta\left(P_{I} \sum_{j=1}^{L_{R}}\left|f_{R, j}\right|^{2}+1\right)\left|h_{k}\right|^{2}+P_{I} \sum_{i=1}^{L_{S_{k}}}\left|f_{S_{k}, i}\right|^{2}+1} .
$$

Remark 1 In this proposed energy harvesting protocol, the channel gains are significant due to exact channel estimation algorithms. For example, the perfect channel state information (CSI) is computed. As a result, the fixed time switching coefficient can be pre-calculated in the energy harvesting phase. However, such coefficient depends on CCIs and the channel gains. A feedback stream is required from relay to source to help the source find the fixed time switching coefficient properly. Note that $\alpha_{1}$ belongs to $[0,1]$.

\section{Performance analysis}

3.1 Signal-to-interference-plus-noise ratio analysis

In this two-way relaying model, $R$ amplifies the received signal $y_{R}$ with a gain $G$ which is expressed as

$$
G^{2}=\left(E_{S}\left|h_{1}\right|^{2}+E_{S}\left|h_{2}\right|^{2}+E_{I} \sum_{j=1}^{L_{R}}\left|f_{R, j}\right|^{2}+N_{0}\right)^{-1}
$$


This AF gain fraction in the high signal-to-interferenceplus-noise ratio (SINR) regime can be expressed again as

$$
G^{2} \approx\left(E_{S}\left(\left|h_{1}\right|^{2}+\left|h_{2}\right|^{2}\right)+E_{I} \sum_{j=1}^{L_{R}}\left|f_{R, j}\right|^{2}\right)^{-1} .
$$

In principle, the transmission of the current signal block at $R$ is provided with enough energy before information is transferred from $R$ to $S_{1}$ and $S_{2}$ in the broadcast phase; it must be sent from $S_{1}$ to $S_{2}$ first. In this paper, the AFbased two-way relaying protocol under the impact of CCI is evaluated in terms of energy harvesting capacity. The performance of SINR of $S_{1}$ is considered.

For simplicity, we only consider the outage performance at one source node, $S_{1}$, in which information is transmitted from $R$ to $S_{1}$ in the broadcast phase, which is expressed as

$$
y_{S_{1}}=G h_{1} \sqrt{E_{S}} y_{R}+\sqrt{E_{I}} \sum_{i=1}^{L_{S_{1}}} f_{S_{1}, i} d_{S_{1}, i}+n_{S_{1}},
$$

and

$$
y_{S_{2}}=G h_{1} \sqrt{E_{S}} y_{R}+\sqrt{E_{I}} \sum_{i=1}^{L_{S_{2}}} f_{S_{2}, i} d_{S_{2}, i}+n_{S_{2}},
$$

where the AWGN components arriving at $S_{1}$ and $S_{2}$ are denoted by $n_{S_{1}}$ and $n_{S_{2}}$, respectively, with mean value $N_{0}$ and $d_{S_{k}, i}$ denotes as interferer signals, $i$ th, to the source $S_{k}$ with unit mean value. It is noted that the average channel coefficients $\Omega_{h_{1}}$ and $\Omega_{h_{2}}$ are known at both sources $S_{1}$ and $S_{2}$ thanks to advanced channel estimation algorithms

$$
\begin{aligned}
y_{S_{1}}= & G h_{1} \sqrt{E_{R}}\left(\sqrt{E_{S}} h_{1} x_{S_{1}}+\sqrt{E_{S}} h_{2} x_{S_{2}}+\sqrt{E_{I}} \sum_{j=1}^{L_{R}} f_{R, j} d_{R, j}+n_{R}\right) \\
& +\sqrt{E_{I}} \sum_{i=1}^{L_{S_{1}}} f_{S_{1}, i} d_{S_{1}, i}+n_{S_{1}},
\end{aligned}
$$

and

$$
\begin{aligned}
y_{S_{2}}= & G h_{2} \sqrt{E_{R}}\left(\sqrt{E_{S}} h_{1} x_{S_{1}}+\sqrt{E_{S}} h_{2} x_{S_{2}}+\sqrt{E_{I}} \sum_{j=1}^{L_{R}} f_{R, j} d_{R, j}+n_{R}\right) \\
& +\sqrt{E_{I}} \sum_{i=1}^{L_{S_{2}}} f_{S_{2}, i} d_{S_{2}, i}+n_{S_{2}},
\end{aligned}
$$

when self-interference cancellation is adopted for the received signal of $S_{1}$ (i.e. their loop self-interference of
$G h_{1} \sqrt{E_{R}} \sqrt{E_{S}} h_{1} x_{S_{1}}$ can be eliminated). For simplicity, the received signal at $S_{1}$ could be expressed as

$$
\begin{aligned}
y_{S_{1}}= & G h_{1} \sqrt{E_{R}} \sqrt{E_{S}} h_{2} x_{S_{2}}+G h_{1} \sqrt{E_{R}} \sqrt{E_{I}} \sum_{j=1}^{L_{R}} f_{R, j} d_{R, j} \\
& +G h_{1} \sqrt{E_{R}} n_{R} \\
& +\sqrt{E_{I}} \sum_{i=1}^{L_{S_{1}}} f_{S_{1}, i} d_{S_{1}, i}+n_{S_{1}}
\end{aligned} .
$$

Thus, the end-to-end SINR $S_{2} \rightarrow R \rightarrow S_{1}$ (it looks similar to the computation for SINR of the link $S_{1} \rightarrow R \rightarrow S_{2}$ ) can be computed by

$$
\gamma_{S_{1}}=\frac{G^{2} E_{R} E_{S}\left|h_{1}\right|^{2}\left|h_{2}\right|^{2}}{G^{2}\left|h_{1}\right|^{2} E_{R}\left(E_{I} \sum_{j=1}^{L_{R}}\left|f_{R, j}\right|^{2}+N_{0}\right)+E_{I} \sum_{i=1}^{L_{S_{1}}}\left|f_{S_{1}, i}\right|^{2}+N_{0}} .
$$

We derive a new expression after simple manipulation which is given by

$$
\gamma_{S_{1}}=\frac{\rho P_{S}\left|h_{1}\right|^{2}\left|h_{2}\right|^{2}}{\rho\left|h_{1}\right|^{2}\left(P_{I} \sum_{j=1}^{L_{R}}\left|f_{R, j}\right|^{2}+1\right)+P_{I} \sum_{i=1}^{L_{S_{1}}}\left|f_{S_{1}, i}\right|^{2}+1} .
$$

It is not complicated to calculate all expressions in terms of SINR. Thus, it is important to achieve approximate expressions for outage probability of $P_{\text {out }}$, which can be expressed as follows:

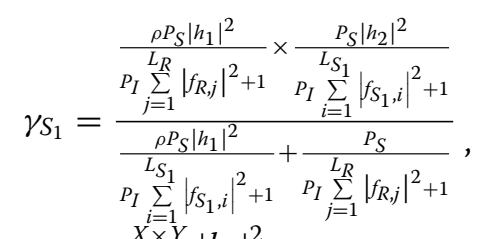

$$
\begin{aligned}
& =\frac{X \times Y}{X+Y}\left|h_{2}\right|^{2}
\end{aligned}
$$

where $X=\frac{\rho P_{S}\left|h_{1}\right|^{2}}{P_{I} \sum_{i=1}^{{ }_{S}}\left|f_{S_{1}, i}\right|^{2}+1}$ and $Y=\frac{P_{S}}{P_{I} \sum_{j=1}^{L_{R}}\left|f_{R, j}\right|^{2}+1}$.

\subsection{Outage probability}

Outage probability performance is considered in Lemma 1.

Lemma $1 X, Y$ denote as arbitrary random variables with $X>1$ and $Y>1$. The tightness of the upper bound is determined as

$$
\frac{X \times Y}{X+Y} \leq \min (X, Y)
$$

Proof We omit it here because it is widely used throughout the paper. 
Nonetheless, in the case of $X=Y$, a new expression is given as

$$
\frac{X \times Y}{X+Y} \leq \min (X, Y)=X=Y .
$$

Determining outage performance based on SINR at (18) is a problem. To the best of our knowledge, the closedform expression of the outage probability in this case does not exist. It motivates us to find a tractable solution. Fortunately, if time switching in the proposed energy harvesting protocol is altered, we derive

$$
\frac{\rho P_{S}\left|h_{1}\right|^{2}}{P_{I} \sum_{i=1}^{L_{S_{1}}}\left|f_{S_{1}, i}\right|^{2}+1}=\frac{P_{S}}{P_{I} \sum_{j=1}^{L_{R}}\left|f_{R, j}\right|^{2}+1} .
$$

As a result, the upper bound of SINR at source $S_{1}$ is achieved

$$
\gamma_{S_{1}, \text { up }}=\frac{P_{S}\left|h_{1}\right|^{2}}{P_{I} \sum_{j=1}^{L_{R}}\left|f_{R, j}\right|^{2}+1} .
$$

As for the threshold rate, $\gamma_{\text {th }}$, the outage probability can be computed in an approximate manner as

$$
\begin{aligned}
P_{\text {out }}\left(\gamma_{\text {th }}\right) \underset{k \in\{1,2\}}{=} F_{\gamma_{S_{k} \text {,up }}}\left(\gamma_{\text {th }}\right) \\
\underset{k \in\{1,2\}}{=} \operatorname{Pr}\left\{\gamma_{S_{k}, \text { up }} \leq 2^{2 R_{S_{0}}}-1 \triangleq \gamma_{\text {th }}\right\}
\end{aligned}
$$

where the threshold rate is denoted by $R_{S_{0}}$.

Proposition 1 In energy harvesting mode, the closedform expression outage probability in an approximate manner at the source $S_{1}$ can be formulated as

$$
\begin{aligned}
P_{\text {out }}\left(\gamma_{\text {th }}\right) & =\operatorname{Pr}\left(\gamma_{S_{1} \text {,up }} \leq \gamma_{\text {th }}\right) \\
& =1-\left(\frac{P_{S} \Omega_{h_{2}} / P_{I} \Omega_{f}}{\gamma_{\text {th }}+P_{S} \Omega_{h_{2}} / P_{I} \Omega_{f}}\right)^{L_{S}} \exp \left(\frac{-\gamma_{\text {th }}}{P_{S} \Omega_{h_{2}}}\right) .
\end{aligned}
$$

Proof See in the Appendix

Furthermore, in the case of no interference, i.e. when $L_{S}=0$, the CDF of $\gamma_{S_{1}}$ can be simplified as

$$
P_{\text {out }}\left(\gamma_{\text {th }}\right)=1-\exp \left(\frac{-\gamma_{\text {th }}}{P_{S} \Omega_{h_{2}}}\right) \text {. }
$$

Interestingly, in high SNR, this assumption is equivalent with high transmit power at the source. Thus, the outage probability can be expressed when $P_{\text {out }}\left(\gamma_{\text {th }}\right)=0$.

\subsection{Throughput in delay-sensitive transmission mode} The instantaneous transmission rate is calculated as

$$
R_{i} \underset{k \in\{1,2\}}{=} \log _{2}\left(1+\gamma_{S_{k}, \text { up }}\right)
$$

By contrast, by evaluating the outage probability, $P_{\text {out }}$, at a fixed source transmission rate, i.e. $R_{S_{0}}(\mathrm{bits} / \mathrm{s} / \mathrm{Hz})$, throughput in the delay-sensitive mode is expressed as

$$
\tau=(1-\alpha) R_{S_{0}}\left(1-P_{\text {out }}\right) .
$$

\subsection{Bit error ratio}

In this subsection, bit error ratio (BER) is considered, in which the error probability of several modulations can be determined by $E\{p Q(\sqrt{2 q \gamma})\}$, where $p$ and $q$ represent modulation-specific constants, SINR is denoted by $\gamma$ and $Q($.) denotes as error function.

The BER can be evaluated using a CDF-based approach which can be calculated as an integral of the CDF of SINR

$$
\mathrm{BER}=\frac{p \sqrt{q}}{2 \sqrt{\pi}} \int_{0}^{\infty} \frac{e^{-q x}}{\sqrt{x}} F_{\gamma_{k_{k}, \text { up }}}(x) d x,
$$

where $F_{\gamma_{S_{k} \text {,up }}}(x)=P_{\text {out }}(x)$. For example, BPSK modulation is investigated as following corresponding parameters $(p=q=1)$.

Based on the expression of error derivation, a mathematical tractable form of BER in specific cases can be expressed by following propositions.

(i) Case 1: CCI

Let us first illustrate the key result as follows:

$$
\left(\frac{1}{A \gamma+1}\right)^{L_{S}}=\frac{A_{1}}{A \gamma+1}+\frac{A_{2}}{(A \gamma+1)^{2}}+\ldots+\frac{A_{L_{S}}}{(A \gamma+1)^{L_{S}}},
$$

in which we denote $A=P_{I} \Omega_{f} / P_{S} \Omega_{h_{2}}$. It is confirmed that a partial fractions decomposition for $\left(\frac{1}{A \gamma+1}\right)^{L_{S}}$ can be easily solved via finding $A_{j}, j=1, \ldots, L_{S}$. Such parameters can be calculated since the fractions in the above equation have the same denominators, following that their numerators must be equal, and then we obtain $A_{j}$.

Proposition 2 The exact form of BER can be expressed as follows:

$$
\begin{aligned}
\mathrm{BER}_{1}= & p \sqrt{\frac{q}{\pi}} \int_{0}^{\infty} \frac{\exp (-q \gamma)}{\sqrt{\gamma}} d \gamma p \sqrt{\frac{q}{\pi}} \sum_{j=1}^{L_{S}} A_{j} \frac{\exp (-q \gamma)}{\sqrt{\gamma}} \\
& \exp \left(\frac{-\gamma}{P_{S} \Omega_{h_{2}}}\right)(A \gamma+1)^{-j} d \gamma
\end{aligned}
$$

and the closed form of BER is

$$
\mathrm{BER}_{1}=p-p \sqrt{\frac{q}{A}} \Psi\left(\frac{1}{2} ; \frac{3}{2}-L_{S} ; \frac{q+\frac{1}{P_{S} \Omega_{h_{2}}}}{A}\right),
$$


where the function $\Psi(x ; y ; z)$ is the confluent hypergeometric function of the second kind, defined by the integral

$$
\Psi(x ; y ; z)=\frac{1}{\Gamma(x)} \int_{0}^{\infty} e^{-z t} t^{x-1}(1+t)^{y-x-1} d t
$$

Proof The closed-form expression of BER in (30) results from substituting (29) into (24) and then applying the BER expression given in (28).

(ii) Case 2: Non-CCI

Interestingly, we henceforth consider the no co-channel interference regime (i.e. $L_{S}=0$ ) and obtain the following results.

Regarding the ideal interference (CCI is approximately 0 ), we can derive a new expression of BER as follows:

Proposition 3 In the special case of non-CCI, the error probability can be significantly simplified to

$$
\begin{aligned}
\mathrm{BER}_{2}= & \frac{p}{2 \sqrt{\pi}} \Phi\left(\frac{1}{2}, q c\right)-\frac{p \sqrt{q}}{2 \sqrt{\pi} \sqrt{q+\frac{1}{P_{S} \Omega_{h 2}}}} \\
& \Phi\left(\frac{1}{2}, q c+\frac{c}{P_{S} \Omega_{h 2}}\right),
\end{aligned}
$$

where $\Phi(m, y)=\int_{0}^{y} t^{m-1} e^{-t} d t$ is the lower incomplete gamma function and $c$ is the threshold SNR.

Proof The closed-form expression of BER in (33) follows from computing (28) using the outage probability in (25).

\section{Simulation results}

In this section, the simulation results are presented to prove the theoretical examinations in terms of outage probability, the average BER and throughput. Note that there is a relay between $S_{1}$ and $S_{2}$, while $d_{1}$ and $d_{2}$ denote the distance between $S_{1}$ and $R$ and $S_{2}$ and $R$, respectively. These distances are normalized as a unit, except in the last simulation as changing such distances satisfies $d_{1}+d_{2}=$ 2. Consequently, $h_{1}$ and $h_{2}$ can be computed by $\Omega_{h_{1}}=d_{1}^{-v}$ and $\Omega_{h_{2}}=d_{2}^{-v}$, respectively, where $v$ represents the path loss exponent which is set to 4 . It is assumed that all channels are flat Rayleigh fading channels, in which elements are independent identically distributed Gaussian random variables, in which the variance is 1 and the mean value is 0 . Meanwhile, flat Rayleigh fading channel coefficients of the interference channels are $\Omega_{f_{R, j}}=\Omega_{f_{S_{k}, i}}=0.7$. Besides that, the equation of power allocation between two terminals and one relay node is $E_{S_{1}} / N_{0}=E_{S_{2}} / N_{0}=$ $E_{S} / N_{0}=P_{S}$. Without loss of generality, it is assumed that $L_{S_{1}}=L_{S_{2}}=L_{S}$.
In Fig. 3, the number of interferers is altered and the approximate equal-power allocation interference case is considered, i.e. $P_{I}$ is a constant value. We set parameters such as $P_{I}=P_{S} / 20(\mathrm{~dB}), \eta=0.4$. This experiment illustrates that the approximate theory results and simulation match well with the accurate results even at a low $P_{S}$ region. Thus, the closed-form approximate formulas provided in this paper are approximately similar to the accurate formulas, and system performance can be evaluated by using such formulas. It can be seen that when the number of interferers increases, the outage probability falls. Moreover, in Fig. 4, outage performance is examined in terms of different values of interference power. Note that if the interference power increases, the outage probability is near the outage limit.

Figure 5 depicts the impact of energy harvesting conversion efficiency on throughput, and numerical results are presented in two cases, interference free and CCI, and the $\mathrm{AF}$ outage performance. Interestingly, if the transmit power increases over a specific limit, e.g. $P_{S}=30 \mathrm{~dB}$, outage performance also remains at both very low harvested power efficiency $(\eta=0.1)$ and medium harvested power efficiency $(\eta=0.4)$.

Figure 6 considers the average BER performance at source $S_{1}$ as a function of a different number of interferers. The BER performance in the case of no interference is evaluated. Furthermore, it is clear that the system performance falls dramatically during the power transmitting period. The performance gap between non-CCI and CCI is obvious.

In Fig. 7, the similar linear trend can be observed, in which the transmission rate is evaluated versus the transmit power of the source node. Note that the instantaneous transmission rate is affected by the interference power.

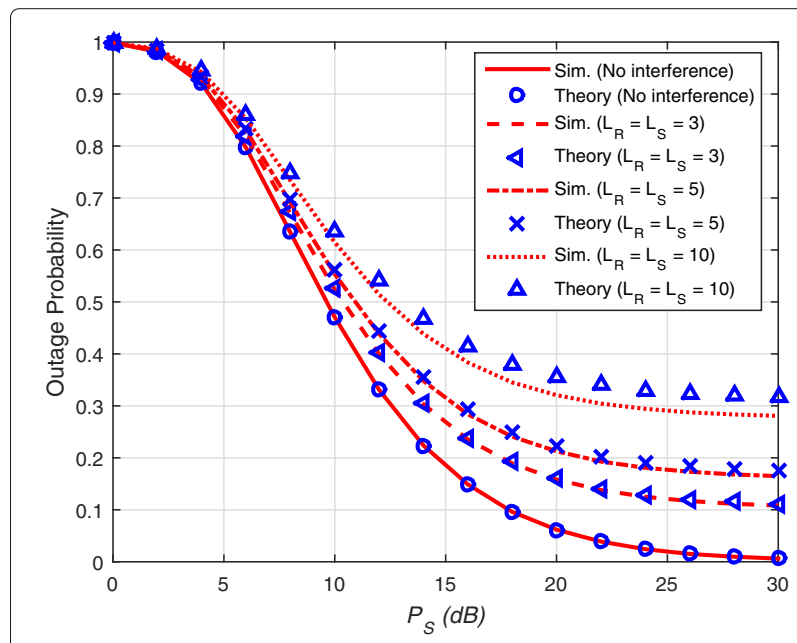

Fig. 3 Outage probability versus the transmit power of the source node with different values of interference power $P_{I}=P_{S} / 20(\mathrm{~dB})$, $\eta=0.4$ 


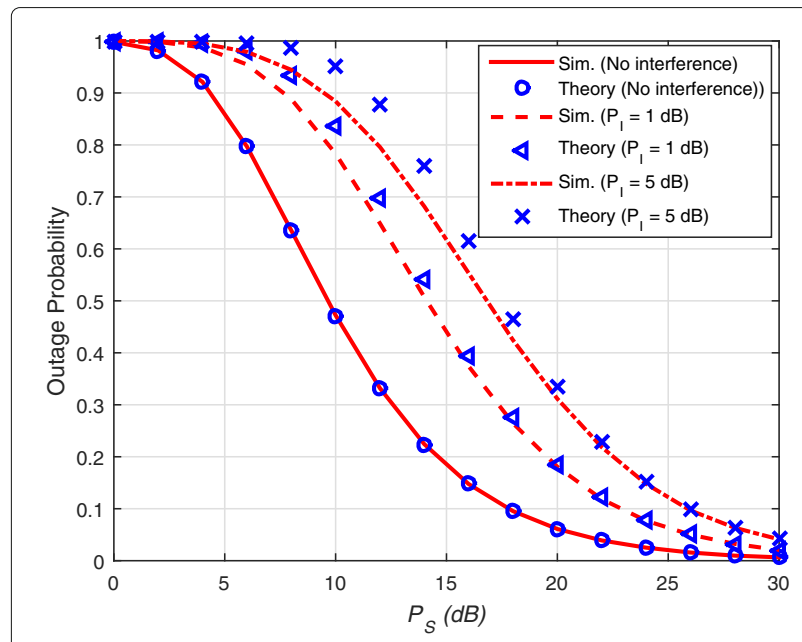

Fig. 4 Outage probability versus the transmit power of the source node with different values of interference power with $L_{S}=L_{R}=3$

Figure 8 considers time allocation in the case of energy harvesting and information processing under non-CCI and CCI cases. Strikingly, the amount of time used for energy harvesting and information processing is equal in an interference-free case. Particularly, as interferer power is high, it increases the harvested energy and reduces time switching fraction.

In the next experiment, we evaluate the impact of physical distance between the source and the relay node on throughput performance as in Fig. 9. This illustration confirmed that the throughput is high when the relay node is closest to the source node. The optimal throughput can be obtained as approximately $d_{1}=0.7$ in the case of

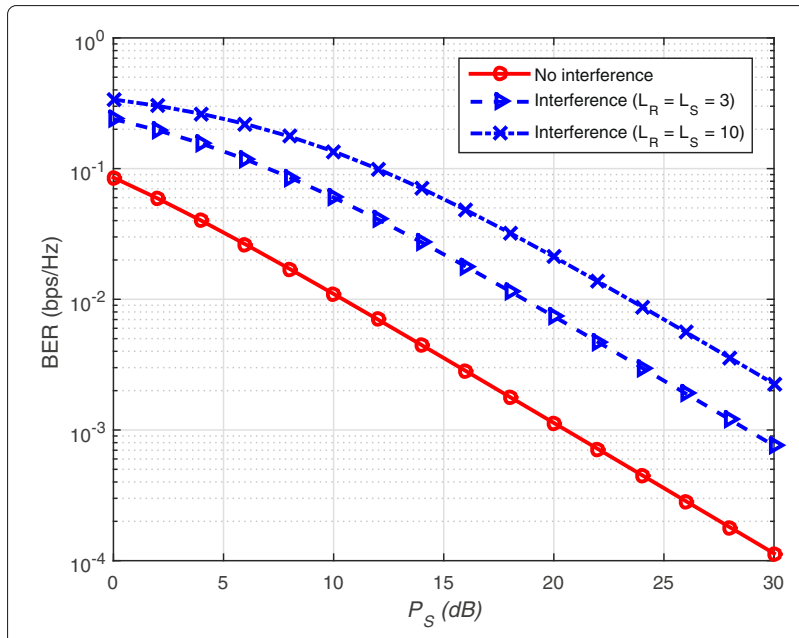

Fig. 6 BER in two-way relaying networks for different values of the transmit power of the source node with $P_{l}=5(\mathrm{~dB})$

CCI and as $d_{1}=0.4$ in the case of non-CCI. Similarly, controlling the interference power level plays an important role in maintaining the system throughput. However, the throughput suddenly changes much by increasing $d_{1}=0.8$ beyond 0.8 . The reason is that the relay node experiences further distance compared with a wireless powered source, which results in fewer values of harvested energy for the remaining reliable communication between the relay and destination nodes. Interestingly, this result is totally different from the traditional case of a nonenergy harvesting-assisted system, in which the maximum throughput is obtained when the position of the relay is between the source and the destination node.

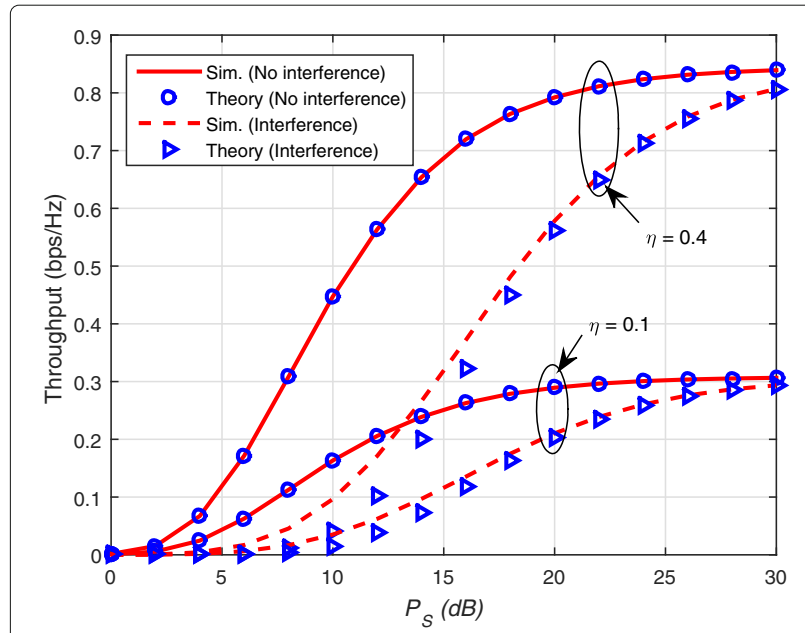

Fig. 5 Comparison of throughput with different values of transmit power of the source node, in which $\eta=0.4$ or $\eta=0.1, L_{S}=L_{R}=3$, $P_{l}=1(\mathrm{~dB})$

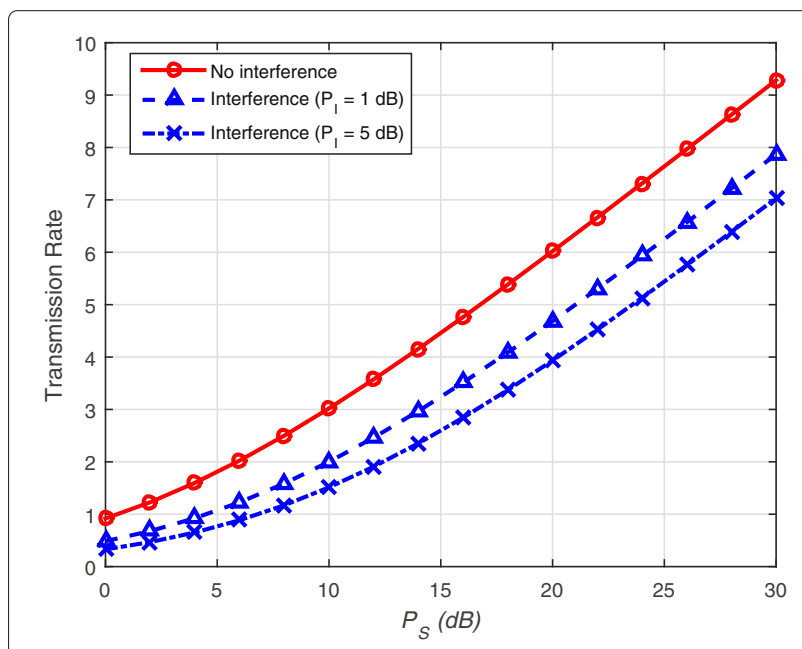

Fig. 7 The instantaneous transmission rate versus different values of transmit power of source node with $L_{S}=L_{R}=3$ 


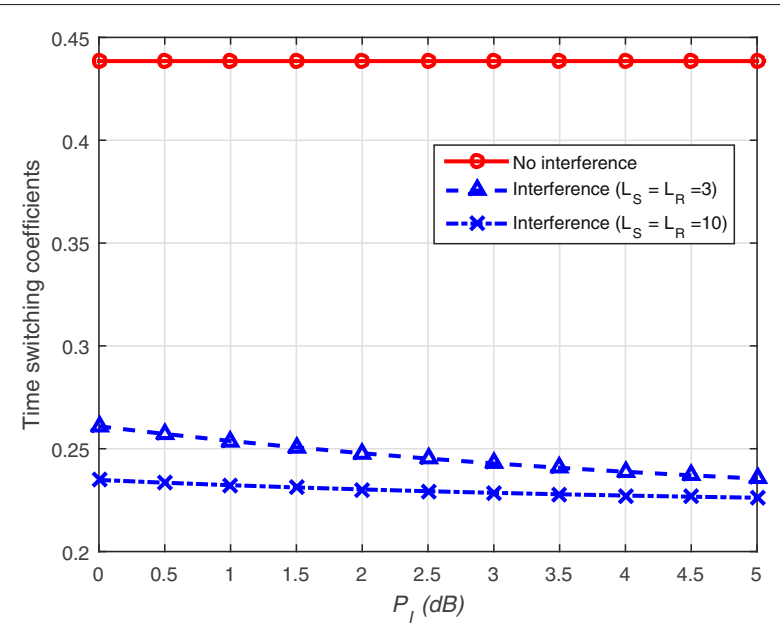

Fig. 8 Time switching coefficients versus the transmit power of interferers

\section{Conclusions}

In this paper, we have carefully analysed RF-based energy harvesting scheme for two-way relaying networks. In particular, the impact of CCI on the performance of AFbased two-way relaying networks is also evaluated for Rayleigh fading channels. When the number of CCIs is altered, we can achieve the exact expressions for SINR and approximate closed-form expressions for outage probability and throughput. Furthermore, to match well with the Monte Carlo simulations in different experiments, analytical expressions are provided. We investigate the impact of CCI and the transmit power of the source node in terms of outage probability and throughput performance to achieve the optimal trade-off. The result is the advantages of energy harvesting in the presence of $\mathrm{CCI}$ and

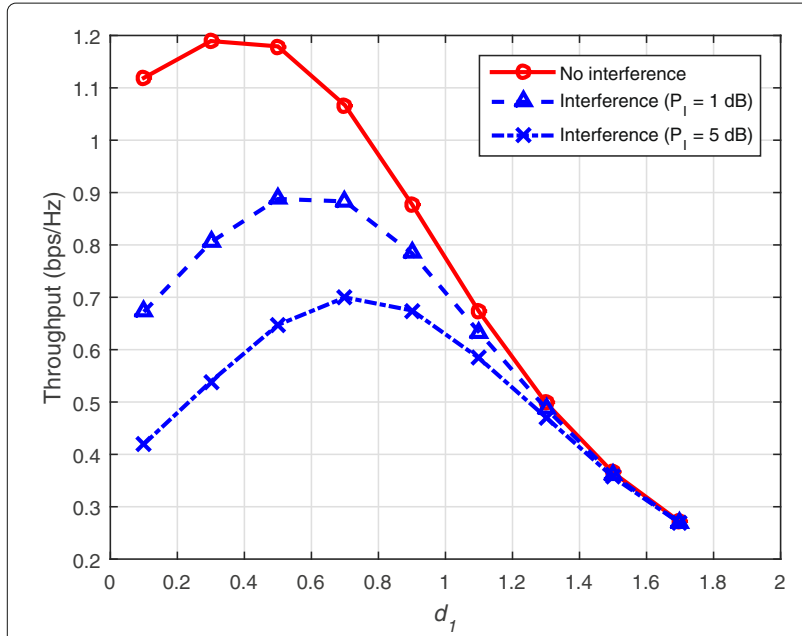

Fig. 9 Throughput performance versus distance between the relay and the source node the parameters, e.g. the number of CCIs and the transmit power of the source which can optimize the throughput and BER performance. Moreover, the closed-form expression of BER in the two-way relaying model considering the presence of $\mathrm{CCI}$ is given.

\section{Appendix}

Proof of Proposition 1 We first rewrite SINR in the following form to obtain the CDF of the upper-bound SINR at node $S_{1}$

$$
\gamma_{S_{1}, \text { up }}=P_{S}\left|h_{1}\right|^{2} /\left(C_{S_{1}}+1\right)
$$

where $C_{S_{1}}=P_{I} \sum_{i=1}^{L_{S_{1}}}\left|f_{S_{1}, i}\right|^{2}$

Next, the PDFs of $P_{S}\left|h_{2}\right|$ and $C_{S_{1}}$ can be written, respectively, as

$$
f_{P_{S}\left|h_{2}\right|}(x)=\left(1 / P_{S} \Omega_{h_{2}}\right) \exp \left(-x / P_{S} \Omega_{h_{2}}\right),
$$

and

$$
f_{C_{S_{1}}}(x)=\left(1 / P_{I} \Omega_{f}\right)^{L_{S_{1}}} \frac{x^{L_{S_{1}}-1}}{\left(L_{S_{1}}-1\right) !} \exp \left(-x / P_{I} \Omega_{f}\right) .
$$

Then, the CDF of SINR is obtained as $[18,21]$

$$
F_{\gamma_{S_{1}, \text { up }}}(\gamma)=1-\left(\frac{P_{S} \Omega_{h_{1}} / P_{I} \Omega_{f}}{\gamma+P_{S} \Omega_{h_{1}} / P_{I} \Omega_{f}}\right)^{L_{S_{1}}} \exp \left(\frac{-\gamma}{P_{S} \Omega_{h_{1}}}\right) \text {. }
$$

This ends the Proof of Proposition 1.

\section{Competing interests}

The authors declare that they have no competing interests.

Received: 12 June 2016 Accepted: 16 November 2016

Published online: 28 November 2016

\section{References}

1. W Lumpkins, Nikola Tesla's dream realized: wireless power energy harvesting. IEEE Consum. Electron. Mag. 3(1), 39-42 (2014). doi:10.1109/MCE.2013.2284940

2. M Pinuela, P Mitcheson, S Lucyszyn, Ambient RF energy harvesting in urban and semi-urban environments. IEEE Trans. Microwave Theory Tech. 61(7), 2715-2726 (2013). doi: 10.1109/TMTT.2013.2262687

3. Dinh-Thuan Do, Optimal throughput under time power switching based relaying protocol in energy harvesting cooperative network. Wirel. Pers. Commun (Springer). 87(2), 551-564 (2016). doi:10.1007/s11277-015-3120-9

4. X Chen, Z Zhang, $\mathrm{H}-\mathrm{H}$ Chen, $\mathrm{H}$ Zhang, Enhancing wireless information and power transfer by exploiting multi-antenna techniques. IEEE Commun. Mag. 53, 133-141 (2015). doi:10.1109/MCOM.2015.7081086

5. LR Varshney, in Proc. IEEE ISIT. Transporting information and energy simultaneously, (Toronto, 2008), pp. 1612-1616. doi:10.1109/ISIT.2008.4595260

6. P Grover, A Sahai, in Proc. IEEE ISIT. Shannon meets Tesla: wireless information and power transfer, (Austin, 2010), pp. 2363-2367. doi:10.1109/isit.2010.5513714

7. R Zhang, C Ho, MIMO broadcasting for simultaneous wireless information and power transfer. IEEE Trans. Wireless Commun. 12(5), 1989-2001 (2013). doi:10.1109/TWC.2013.031813.120224 
8. A Agustin, JVidal, Amplify-and-forward cooperation under interference-limited spatial reuse of the relay slot. IEEE Trans. Wireless Commun. 7(5), 1952-1962 (2008). doi:10.1109/TWC.2008.070973

9. I Krikidis, JS Thompson, S McLaughlin, N Goertz, Max-min relay selection for legacy amplify-and-forward systems with interference. IEEE Trans. Wireless Commun. 8(6), 3016-3027 (2009). doi:10.1109/TWC.2009.080383

10. A Bletsas, AG Dimitriou, JN Sahalo, Interference-limited opportunistic relaying with reactive sensing. IEEE Trans. Wireless Commun. 9(1), 14-20 (2010). doi:10.1109/TWC.2010.01.081128

11. C Zhong, S Jin, K-K Wong, Dual-hop systems with noisy relay and interference-limited destination. IEEE Trans.Commun. 58(3), 764-768 (2010). doi:10.1109/TCOMM.2010.03.080156

12. Y Gu, S Aissa, in Proc. IEEE International Conference on Communications (ICC'14). Interference-aided energy harvesting in decode-and-forward relaying systems, (Sydney, 2014), pp. 5389-5393. doi:10.1109/ICC.2014.6884176

13. $\mathrm{K}$ Song, $\mathrm{B} \mathrm{Ji}$, Y Huang, M Xiao, L Yang, Performance analysis of antenna selection in two-way relay networks. IEEE Trans.Signal Process. 63(10), 2520-2532 (2015). doi:10.1109/TSP.2015.2414904

14. T Soithong, VA Aalo, GP Efthymoglou, C Chayawan, Performance of multi-hop relay systems with co-channel interference in Rayleigh fading channels. IEEE Commun. Lett. 15(8), 836-838 (2011). doi:10.1109/LCOMM.2011.062211.110747

15. S Ikki, S Assa, Multi-hop wireless relaying systems in the presence of cochannel interferences: performance analysis and design optimization. IEEE Trans. Veh. Technol. 61(2), 566-573 (2012). doi:10.1109/TVT.2011.2179818

16. T Soithong, VA Aalo, GP Efthymoglou, C Chayawan, Outage analysis of multihop relay systems in interference-limited Nakagami-m fading channels. IEEE Trans. Veh. Technol. 61(3), 1451-1457 (2012). doi:10.1109/TVT.2012.2185525

17. M Wen, X Cheng, A Huang, B Jiao, Asymptotic performance analysis of multihop relaying with co-channel interference in Nakagami-m fading channels. IEEE Commun. Lett. 16(9), 1450-1453 (2012) note=doi:10.1109/LCOMM.2012.071612.120958,

18. I Trigui, S Affes, A St'ephenne, Ergodic capacity analysis for interference-limited AF multi-hop relaying channels in Nakagami-m fading. IEEE Trans. Commun. 61(7), 2726-2734 (2013). doi:10.1109/TCOMM.2013.052013.120900

19. AA Nasir, Z Xiangyun, S Durrani, Relaying protocols for wireless energy harvesting and information processing. IEEE Trans. Wireless Commun. 12(7), 3622-3636 (2013). doi:10.1109/TWC.2013.062413.122042

20. R Zhao, Y Huang, W Wang, V Lau, Ergodic achievable secrecy rate of multiple-antenna relay systems with cooperative jamming. IEEE Trans. Wireless Commun. 15(4), 2537-2551 (2016). doi:10.1109/TWC.2015.2504526

21. A Papoulis, SU Pillai, Random Variables and Stochastic Processes, 4th Ed. (McGraw Hill, 2002)

\section{Submit your manuscript to a SpringerOpen ${ }^{\circ}$ journal and benefit from:}

- Convenient online submission

- Rigorous peer review

- Immediate publication on acceptance

- Open access: articles freely available online

- High visibility within the field

- Retaining the copyright to your article

Submit your next manuscript at $\gg$ springeropen.com 